NASA Technical Memorandum 106046

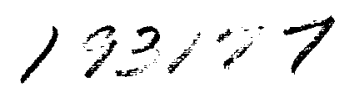

Finite Element Analysis of Structural Engineering Problems Using a Viscoplastic Model Incorporating Two Back Stresses

Vinod K. Arya

University of Toledo

Toledo, Ohio

and

Gary R. Halford

Lewis Research Center

Cleveland, Ohio

October 1993

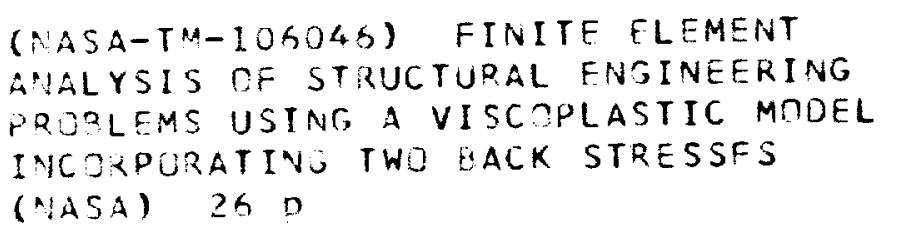

N94-16881

Unclas 



\title{
FINITE ELEMENT ANALYSIS OF STRUCTURAL ENGINEERING
}

\author{
PROBLEMS USING A VISCOPLASTIC MODEL \\ INCORPORATING TWO BACK STRESSES \\ Vinod K. Arya ${ }^{*}$ \\ University of Toledo \\ Toledo, Ohio 43606 \\ and \\ Gary R. Halford \\ National Aeronautics and Space Administration \\ Lewis Research Center \\ Cleveland, Ohio 44135
}

\section{ABSTRACT}

The feasibility of a viscoplastic model incorporating two back stresses and a drag strength is investigated for performing nonlinear finite element analyses of structural engineering problems. The model has been recently put forth by Freed and Walker. To demonstrate suitability for nonlinear structural analyses, the model is implemented into a finite element program and analyses for several uniaxial and multiaxial problems are performed. Good agreement is shown between the results obtained using the finite element implementation and those obtained experimentally. The advantages of using advanced viscoplastic models for performing nonlinear finite element analyses of structural components are indicated.

\section{INTRODUCTION}

Classical creep-plasticity constitutive models treat creep and plastic strains as independent noninteracting entities. These models are, therefore, incapable of

\footnotetext{
${ }^{*}$ NASA Resident Research Associate at Lewis Research Center.
} 
accounting for the observed interactions between creep and plastic strains at high temperatures. Viscoplastic models, however, consider all the inelastic strain (including plasticity, creep, relaxation, etc.) as a single, unified, time-dependent quantity, and thus, automatically include interactions that occur among them. Viscoplastic models, therefore, provide more realistic descriptions of timedependent inelastic behavior of materials at high temperatures.

Viscoplastic models become more realistic when as much material physics as possible is included in the model. This, however, results in complex mathematical frameworks for viscoplastic models. The constitutive differential equations of viscoplastic models that govern the flow and evolution laws are generally highly nonlinear and mathematically "stiff." The closed-form solutions for structural engineering problems are virtually intractable when viscoplastic models are used to define the stress-strain relationship. To assess the advantages offered by more realistic viscoplastic models one must, therefore, employ numerical solution methodology involving, for example, the finite element method or the boundary element method.

Toward this aim, this paper presents a finite element solution methodology developed at the National Aeronautics and Space Administration (NASA) Lewis Research Center for use with the general purpose finite element program MARC [MARC Analysis Research Corporation, 1992]. The methodology, designed for use with viscoplastic models, is demonstrated in this paper for a viscoplastic model recently developed by Freed and Walker [1993] that incorporates two back 
stresses. However, because the methodology is general in nature, it can easily be adapted for use with other viscoplastic models.

For completeness, the paper includes brief descriptions of the viscoplastic model by Freed and Walker [1993] and the finite element solution methodology. The methodology is illustrated by applying it to several uniaxial and multiaxial problems.

\section{VISCOPLASTIC MODEL}

The viscoplastic model used herein was recently put forth by Freed and Walker [1993]. The model contains one scalar internal state variable $\mathrm{D}$, called the drag strength, and a tensorial internal variable $B_{\mathrm{ij}}$, called the back stress. The back stress $B_{i j}$ is assumed to be composed of two back stresses that are denoted by $B_{\mathrm{ij}}^{\mathrm{s}}$ and $\mathrm{B}_{\mathrm{ij}}^{\ell}$. ( $A$ small displacement and a small strain formulation is employed in the model.)

The stress $\sigma_{\mathrm{ij}}$ is taken to be related to elastic strain by Hooke's law

$$
\varepsilon_{\mathrm{ij}}^{\mathrm{e}}=\frac{1+\nu}{\mathrm{E}} \sigma_{\mathrm{ij}}-\frac{\nu}{\mathrm{E}} \sigma_{\mathrm{kk}} \delta_{\mathrm{ij}} \quad(\mathrm{i}, \mathrm{j}=1,2,3)
$$

where $\mathrm{E}$ is Young's modulus, $\nu$ is Poisson's ratio, and $\delta_{\mathrm{ij}}$ is the Kronecker delta. The symbol $\varepsilon$ denotes the strain and superscript e denotes the elastic component of strain. Following Einstein's summation convention, the repeated subscripts in equation (1) and elsewhere imply summation over the range of the symbol.

The total strain rate $\dot{\varepsilon}_{\mathrm{ij}}$ is written as the sum of elastic $\dot{\varepsilon}_{\mathrm{ij}}^{\mathrm{e}}$, inelastic (including plasticity, creep, relaxation, etc.) $\dot{\varepsilon}_{\mathrm{ij}}^{\mathrm{v}}$, and thermal $\dot{\varepsilon}_{\mathrm{ij}}^{\mathrm{t}}$ strain rate components. In symbol form, 
(2)

$$
\dot{\varepsilon}_{\mathrm{ij}}=\dot{\varepsilon}_{\mathrm{ij}}^{\mathrm{e}}+\dot{\varepsilon}_{\mathrm{ij}}^{\mathrm{v}}+\dot{\varepsilon}_{\mathrm{ij}}^{\mathrm{t}} \quad(\mathrm{i}, \mathrm{j}=1,2,3)
$$

In equation (2) the superscripts $\mathrm{v}$ and $\mathrm{t}$ denote the viscoplastic and thermal components of strain rate, respectively. A dot over a symbol indicates its derivative with respect to time $t$.

The deviatoric stress $\mathrm{S}_{\mathrm{ij}}$ and the deviatoric total strain rate $\dot{\mathrm{E}}_{\mathrm{ij}}$ have the following expressions

$$
\mathrm{S}_{\mathrm{ij}}=\sigma_{\mathrm{ij}}-\frac{1}{3} \sigma_{\mathrm{kk}} \delta_{\mathrm{ij}}
$$

and

$$
\dot{\mathrm{E}}_{\mathrm{ij}}=\dot{\varepsilon}_{\mathrm{ij}}-\frac{1}{3} \dot{\varepsilon}_{\mathrm{kk}} \delta_{\mathrm{ij}}
$$

The back stress $B_{i j}$ is the sum of two back stress components $B_{i j}^{s}$ and $B_{i j}^{\ell}$, that is

$$
B_{i j}=B_{i j}^{s}+B_{i j}^{\ell}
$$

The effective stress $\Sigma_{\mathrm{ij}}$ is defined as

$$
\Sigma_{\mathrm{ij}}=\mathrm{S}_{\mathrm{ij}}-\mathrm{B}_{\mathrm{ij}}
$$

The temperature dependence of the model is mainly contained in the thermal diffusivity function $\Theta$, which is defined as

$$
\Theta= \begin{cases}\exp (-\mathrm{Q} / \mathrm{kT}) & \mathrm{T}_{\mathrm{t}} \leq \mathrm{T} \leq \mathrm{T}_{\mathrm{m}} \\ \exp \left[-\frac{\mathrm{Q}}{\mathrm{kT}}\left\{\ln \left(\frac{\mathrm{T}_{\mathrm{t}}}{\mathrm{T}}\right)+1\right\}\right] & 0 \leq \mathrm{T} \leq \mathrm{T}_{\mathrm{t}}\end{cases}
$$


In equation (7), $Q$ is the activation energy, $k$ is the Boltzmann constant, $T$ is absolute temperature, $T_{t}$ is transition temperature, and $T_{m}$ is the melting temperature of the material.

The function $\mathrm{Z}$, the Zener-Hollomon parameter, is defined by

$$
\mathrm{Z}=\mathrm{A} \sinh ^{\mathrm{n}}\left(\sqrt{\mathrm{J}_{2}} / \mathrm{D}\right)
$$

where

$$
\mathrm{J}_{2}=\Sigma_{\mathrm{ij}} \Sigma_{\mathrm{ij}} / 2
$$

Here $A$ and $n$ are inelastic material constants.

The evolution equations for the internal state variables are

$$
\dot{\mathrm{B}}_{\mathrm{ij}}^{\alpha}=2 \mathrm{H}_{\alpha}\left(\dot{\bar{\varepsilon}}_{\mathrm{ij}}^{\mathrm{v}}-\frac{\mathrm{B}_{\mathrm{ij}}^{\alpha}}{2 \mathrm{~L}_{\alpha}} \mathrm{I}_{2}\right)
$$

where $\alpha=\mathrm{s}, \ell$. The strain invariant $\mathrm{I}_{2}$ is defined by

$$
I_{2}=\left\{\begin{array}{lll}
\Theta Z & \text { if } & J_{2}<K^{2} \\
P_{i j} \dot{E}_{i j} / P^{M} & & \text { otherwise }
\end{array}\right.
$$

In equation (10)

$$
P_{i j}=\frac{E}{2(1+\nu)} \frac{\Sigma_{i j}}{\sqrt{J_{2}}}
$$

and $\dot{\mathrm{E}}_{\mathrm{ij}}$ is defined by equation (4). Also

$$
\mathrm{P}^{\mathrm{M}}=\frac{\mathrm{E}}{2(1+\nu)}+\Sigma_{\alpha} \frac{\mathrm{H}_{\alpha}}{\mathrm{L}_{\alpha}}\left(\mathrm{L}_{\alpha}-\frac{1}{2} \mathrm{~B}_{\mathrm{ij}}^{\alpha} \frac{\Sigma_{\mathrm{ij}}}{\sqrt{\mathrm{J}_{2}}}\right)
$$

The functions $\mathrm{K}, \mathrm{H}_{\alpha}$, and $\mathrm{L}_{\alpha}$ in the above equations are given by

$$
\mathrm{K}=\left(\mathrm{C}-\mathrm{D}_{0}\right)\left(\mathrm{C}+\mathrm{D}_{0}\right) / 4 \delta \mathrm{C}
$$




$$
\mathrm{H}_{\mathrm{s}}=\frac{\mathrm{E}}{2(1+\nu)} \quad \mathrm{H}_{\mathrm{l}}=\frac{\mathrm{E}}{2(1+\nu) \mathrm{H}_{0}}
$$

and

$$
\mathrm{L}_{\mathrm{s}}=\mathrm{f} \frac{(\mathrm{C}-\mathrm{D})\left(\mathrm{D}-\mathrm{D}_{0}\right)}{\delta \mathrm{C}} \quad \mathrm{I}_{\ell}=(1-\mathrm{f}) \frac{(\mathrm{C}-\mathrm{D})\left(\mathrm{D}-\mathrm{D}_{0}\right)}{\delta \mathrm{C}}
$$

in which $\mathrm{C}, \delta, \mathrm{H}_{0}$, and $\mathrm{f}$ are material constants and $\mathrm{D}_{0}$ denotes the minimum value that the drag strength $\mathrm{D}$ can take for a given material.

The drag strength $\mathrm{D}$ evolves according to the following equation

$$
\dot{\mathrm{D}}=\mathrm{h}\left(\mathrm{I}_{2}-\Theta \mathrm{r}\right) \quad \mathrm{D}_{0} \leq \mathrm{D} \leq \mathrm{D}_{\max }
$$

where

$$
\mathrm{D}_{\max }=\left(\mathrm{C}+\mathrm{D}_{0}\right) / 2
$$

and

$$
\mathbf{r}=\mathbf{A} \sinh \left[\left(\mathrm{D}-\mathrm{D}_{0}\right) / \delta \mathrm{C}\right]^{\mathrm{n}}
$$

The function $\mathrm{h}$ is defined as

$$
\mathrm{h}=\mathrm{h}_{\mathrm{D}}\left[\frac{\left(\mathrm{D}-\mathrm{D}_{0}\right) / \delta \mathrm{C}}{\sinh \left\{\left(\mathrm{D}-\mathrm{D}_{0}\right) / \delta \mathrm{C}\right\}}\right]^{\mathrm{m}}
$$

where

$$
h_{D}=\left\{\begin{array}{lc}
h_{1} & T_{t} \leq \mathrm{T} \leq \mathrm{T}_{\mathrm{m}} \\
\mathrm{h}_{0}-\frac{\mathrm{h}_{0}-\mathrm{h}_{1}}{\mathrm{~T}_{\mathrm{t}}} \mathrm{T} & 0<\mathrm{T} \leq \mathrm{T}_{\mathrm{t}}
\end{array}\right.
$$

In equation (18), $h_{0}$ and $h_{1}$ are material constants.

In addition to elastic material constants, the shear modulus $\mu$, Poisson's ratio $\nu$, and the coefficient of thermal expansion $\alpha$, the above viscoplastic model has 13 inelastic material constants: $A, C, \delta, D_{0}, f, H_{0}, h_{0}, h_{1}, m, n, Q, T_{m}$, and 
$T_{t}$. The values of these constants taken from Freed and Walker [1993] are listed in Table I.

\section{DESCRIPTION OF MARC PROGRAM}

A viscoplastic model is most advantageous if it can be used on a practical scale for the solution of structural engineering problems faced by, for example, the aerospace and nuclear industries. To demonstrate the feasibility of the present viscoplastic model for performing complex nonlinear structural analyses, the model was implemented into the general purpose finite element program MARC [1992]. Any other finite element program capable of performing nonlinear viscoplastic structural analyses can also be used. The MARC program employs sophisticated integration algorithms and advanced finite element formulations. It is specially tailored to fit the requirements of a nonlinear structural analysis.

The MARC program provides the user with a convenient way of implementing and integrating the constitutive differential equations of Freed and Walker's model [F \& W, 1993]. This is accomplished through the user subroutine HYPELA [see MARC Analysis Research Corporation, 1992] provided in MARC. The subroutine HYPELA, developed for Freed and Walker's model, as well as the self-adaptive time integration strategy used in it, follow essentially the same structure as given in Arya and Kaufman [1989] and Cassenti [1983].

In the MARC program, all the material nonlinearities are incorporated into an initial load vector that is treated as a pseudo-body force in finite element equilibrium equations. The constitutive equations of viscoplastic models are 
mathematically "stiff." A subincrement technique is employed to form the incremental constitutive equations corresponding to a given finite load increment.

In the subincrement technique, a given finite load increment is split into several equal subincrements. The constitutive equations of the viscoplastic models are then integrated over these small subincrements to obtain an accurate representation of the incremental constitutive equations over the finite load increment. Structural engineering problems encountered in aerospace and nuclear industries usually involve many degrees of freedom. Since the use of an explicit integration method does not require the assembly and inversion of Jacobian matrices, it is more suitable for problems involving a large number of degrees of freedom. The explicit forward Euler method with a self-adaptive time step was used to integrate the constitutive equations over the subincrements. The integration strategy is found to work efficiently and accurately, even for large finite element load increments, provided the subincrements are suitably small to ensure the stability of the forward Euler method (see, for example, Arya [1992] and Arya and Arnold [1992]). However, it is difficult for the user to select efficient subincremental steps, and there is a considerable incentive (saved CPU time) to use as few subincrements as possible without affecting the stability of the constitutive equations associated with the viscoplastic model. For an experienced user, however, it is easier to optimize the number of subincrements.

A brief introduction to the MARC program is provided here to familiarize the user with its operation. (For complete details of the MARC program, see 
MARC Analysis Research Corporation, 1992.) This introduction, taken from Walker [1981], is as follows:

"The principle of virtual work may be used to generate the MARC non-linear equilibrium equations governing the incremental response of the structure to an increment of load. In evaluating the non-linear structural response of a component, the program assumes that the load history is divided into incrementally applied loads.

Each load step is sequentially analyzed as a linear matrix problem using an appropriate stiffness matrix and load vector. Although each load step uses linear matrix methods to solve the incremental equations, the incremental equations themselves are non-linear since the load vector will depend on the displacement increment obtained in the solution of incremental equilibrium equations.

The principle of virtual work may be written, for applied external point loads $P_{i}$, or displacement $u_{i}$, in the form:

$$
\Sigma \int_{V} \delta \varepsilon_{i}^{\mathrm{T}} \sigma_{\mathrm{i}} \mathrm{dV}=\delta \mathrm{u}_{\mathrm{i}}^{\mathrm{T}} \mathrm{P}_{\mathrm{i}}
$$

where the integral extends over the volume, V, of each finite element and the summation sign extends to all the elements in structure.

In Eq. (5.1) the virtual displacement vector $\delta \mathrm{u}_{\mathrm{i}}$ is related to the virtual strain vector $\delta \varepsilon_{\mathrm{i}}$ through the relationship: 


$$
\delta \varepsilon_{\mathrm{i}}=\mathrm{B}_{\mathrm{ij}} \delta_{\mathrm{uj}} \quad \text { so that } \quad \delta \varepsilon_{\mathrm{i}}^{\mathrm{T}}=\delta_{\mathrm{uj}}^{\mathrm{T}} \mathrm{B}_{\mathrm{ij}}^{\mathrm{T}}
$$

where $B_{i j}$ is the strain displacement matrix and the superscript $T$ denotes transposition. Since $\delta u_{i}$ is an arbitrary virtual displacement vector, Eqs. (5.1) and (5.2) may be written in the form:

$$
\Sigma \int_{V} B_{i j}^{T} \sigma_{j} d V=P_{i}
$$

This relation expresses the equilibrium of structure when the applied load vector is $P_{i}$ and the stress vector is $\sigma_{\mathrm{i}}$. If an incremental load $\Delta \mathrm{P}_{\mathrm{i}}$ is applied to the structure and the stress vector changes to $\sigma_{\mathrm{i}}+\Delta \sigma_{\mathrm{i}}$, the relation expressing the equilibrium of the structure at the end of the incremental load application may be written as:

$$
\Sigma \int_{V} B_{i j}^{T}\left(\sigma_{j}+\Delta \sigma_{j}\right) d V=P_{i}+\Delta P_{i}
$$

Hence, the relation expressing the equilibrium of the structure during the application of the incremental load vector $\Delta \mathrm{P}_{\mathrm{i}}$ is obtained from Eqs. (5.3) and (5.4) by subtraction in the form:

$$
\Sigma \int_{\mathrm{V}} \mathrm{B}_{\mathrm{ij}}^{\mathrm{T}} \Delta \sigma_{\mathrm{j}} \mathrm{dV}=\Delta \mathrm{P}_{\mathrm{i}}
$$

The MARC code allows the user to implement very general constitutive relationships into the program by means of the user subroutine HYPELA. Within this subroutine, the user must specify the values of elasticity matrix $D_{i j}$ and the inelastic stress vector $\Delta \zeta_{\mathrm{i}}$ in the incremental vector constitutive relationship: 


$$
\Delta \sigma_{\mathrm{i}}=\mathrm{D}_{\mathrm{ij}}\left(\Delta \varepsilon_{\mathrm{j}}-\delta_{\mathrm{j}} \alpha \Delta \theta\right)-\Delta \zeta_{\mathrm{i}}
$$

The inelastic stress increment vector $\Delta \zeta_{i}$ is computed in HYPELA using the constitutive relationships ... [of the viscoplastic model].

In Eq. (5.6), $\alpha$ denotes the coefficient of thermal expansion and $\delta_{\mathrm{j}}$ is the vector Kronecker delta symbol,

$$
\delta_{\mathrm{j}}=\left\{\begin{array}{lll}
1 & \text { if } & 0 \leq \mathrm{j} \leq 3 \\
0 & \text { if } & 3 \leq \mathrm{j} \leq 6
\end{array}\right.
$$

For the ... [viscoplastic models], the incremental inelastic stress vector $\Delta \zeta_{\mathrm{i}}$ depends in a highly nonlinear manner on the incremental strain vector $\Delta \varepsilon_{\mathrm{j}}$. Since $\Delta \varepsilon_{\mathrm{j}}=\mathrm{B}_{\mathrm{ij}} \Delta_{\mathrm{uj}}$, the incremental inelastic stress vector $\Delta \zeta_{\mathrm{i}}$ depends in a highly non-linear manner on the nodal displacement vector $\Delta_{\mathrm{uj}}$, so that $\Delta \zeta_{\mathrm{i}}=\Delta \zeta\left(\Delta_{\mathrm{uj}}\right)$. Substitution of Eq. (5.6) into (5.5) produces the incremental equilibrium equations for MARC program in the form:

$$
\Sigma \mathrm{K}_{\mathrm{ij}} \Delta \mathrm{u}_{\mathrm{j}}=\Delta \mathrm{P}_{\mathrm{i}}+\Delta \mathrm{R}_{\mathrm{i}}+\Sigma \int_{\mathrm{V}} \mathrm{B}_{\mathrm{ij}}^{\mathrm{T}} \Delta \zeta_{\mathrm{j}} \mathrm{dV}+\Sigma \int_{\mathrm{V}} \mathrm{B}_{\mathrm{ij}}^{\mathrm{T}} \delta_{\mathrm{j}} \alpha \Delta \theta \mathrm{dV}
$$

where $\mathrm{K}_{\mathrm{ij}}$ is the elemental elastic stiffness matrix defined by the relation:

$$
K_{i j}=\int_{V} B_{i k}^{T} D_{k \ell} B_{\ell j} d V
$$

The vector $\Delta \mathrm{R}_{\mathrm{i}}$ is the residual load correction vector or out-ofequilibrium force vector from the preceding load increment:

$$
\Delta R_{i}=P_{i}-\Sigma \int_{V} B_{i j} \sigma_{j} d V
$$


which is added to the current increment in order to restore the structure to equilibrium. The nonlinearity in the incremental equilibrium relationship, defined in Eq. (5.8), arises because the inelastic stress increment vector $\Delta \zeta_{\mathrm{i}}$ depends non-linearly on the displacement increment vector $\Delta \mathrm{u}_{\mathrm{j}}$. Values of $\mathrm{D}_{\mathrm{ij}}$ and $\Delta \zeta_{\mathrm{j}}$ appropriate to the current incremental load step are returned to the main program by subroutine HYPELA and the incremental equilibrium relations in Eq. (5.8) are solved by successive iterations.

The solution of the incremental equilibrium equations in (5.8) is accomplished within the MARC code by the following algorithm. At the start of the increment the user subroutine HYPELA is entered to determine the elasticity matrix $D_{i j}$ and the incremental inelastic stress vector $\Delta \zeta_{\mathrm{i}}$. On entry to the subroutine, the input consists of the strain increment vector $\Delta \varepsilon_{\mathrm{j}}$, the temperature increment $\Delta \Theta$, the time increment $\Delta$ t over which the incremental external load vector $\Delta \mathrm{P}_{\mathrm{i}}$ is applied to the structure, and the values of stress, strain, temperature and viscoplastic state variables at the beginning of the increment. Since the incremental strain vector $\Delta \varepsilon_{\mathrm{i}}=\mathrm{B}_{\mathrm{ij}} \Delta_{\mathrm{uj}}$, can only be accurately determined after the solution to the incremental equilibrium relationship in Eq. (5.8) has yielded the correct incremental solution vector $\Delta_{\mathrm{uj}}$, the strain increment vector $\Delta \varepsilon_{\mathrm{i}}$ initially used to generate the inelastic stress increment vector $\Delta \zeta_{\mathrm{i}}$ must be estimated. The initial estimate for $\Delta \varepsilon_{\mathrm{i}}$ is 
assumed to be the value obtained for $\Delta \varepsilon_{\mathrm{i}}$ in the preceding increment. On exit from subroutine HYPELA the elasticity matrix $D_{i j}$ and the estimated inelastic stress increment vector $\Delta \zeta_{\mathrm{i}}$ are passed into the main program. After the values of $D_{i j}$ and $\Delta \zeta_{i}$ are obtained for each integration point in the structure, the incremental equilibrium relationship in Eq. (5.8) is assembled and solved for the incremental node displacement vector $\Delta_{\mathrm{uj}}$. The incremental strain vector, $\delta \varepsilon_{\mathrm{i}}=\mathrm{B}_{\mathrm{ij}} \Delta_{\mathrm{uj}}$, is then computed and compared with the initial guess for $\Delta \varepsilon_{\mathrm{i}}$ used to generate the inelastic incremental stress vector $\Delta \zeta_{\mathrm{j}}$. If this incremental strain vector is equal, within a user specified tolerance, to the incremental strain vector used to compute $\Delta \zeta_{\mathrm{j}}$ in the assembly phase, the solution is assumed to have converged. Otherwise the updated strain increment vector, obtained from the solution of the equilibrium relations in Eq. (5.8), is passed into subroutine HYPELA, a new vector, $\Delta \zeta_{j}$, is computed and the equilibrium equations resolved to yield an improved value of $\Delta_{\mathrm{uj}}$ and $\Delta \varepsilon_{\mathrm{i}}$. The process is repeated until the value of vector $\Delta \varepsilon_{\mathrm{i}}$ on the assembly phase is equal, within a user specified tolerance, to the value of vector $\Delta \varepsilon_{\mathrm{i}}$ on the solution phase. After convergence is achieved, the temperature, stress vector, strain vector and viscoplastic state variables are updated by adding the incremental values generated during the current increment to the values of these variables at the beginning of the increment. The 
program then passes on to the next load increment where the process is repeated."

\section{APPLICATION TO PROBLEMS}

\subsection{Uniaxial Problems}

Some uniaxial problems were solved first to demonstrate and validate the implementation of Freed and Walker's model into the MARC program. These included calculations of tensile stress-strain response at different temperatures and cyclic response under isothermal and nonisothermal loadings. The constitutive equations (including the flow and evolution laws) of the viscoplastic model were implemented in MARC in their generalized three-dimensional form through the user subroutine HYPELA. This generalized finite element implementation was used to perform the uniaxial computations that are presented in this paper. The values of constants for copper listed in Table I and taken from Freed and Walker [1993], were utilized in these computations.

Figures 1 through 4 show the results for uniaxial problems.

\subsection{Multiaxial Cowl Lip Problem}

To demonstrate the feasibility of performing complex structural analyses using a viscoplastic model, the validated finite element implementation of Freed and Walker's viscoplastic model was applied to a sample structural component used in the aerospace industry. The component, called a cowl lip, is part of the leading edge of a hypersonic aircraft engine inlet. To achieve high inlet aerodynamic performance for proposed hypersonic flight between Mach 3 and 25, not only must the high heat flux and high heating rates be tolerated, but 
distortions caused by thermal warping of the structure must also be minimized. Consequently, the need arises for the development of actively cooled leading edges fabricated from specialized materials as well as innovative cooling concepts that enable a structure to withstand the severe thermal loading conditions. The details of different cooling concepts proposed under a NASA Lewis sponsored program, called COLT (Cowl Lip Technology Program), were presented by Melis and Gladden [1988]. In this paper, a cooling concept, called the parallel flow concept, is investigated. In this concept, the coolant channels are laid parallel to the leading edge of the cowl lip (fig. 5), and the coolant is flowed through them to contain the temperature of the component. The viability of the parallel flow concept is investigated by performing the structural analysis with the finite element implementation of Freed and Walker's viscoplastic model.

The cowl lip geometry is shown in figure 5. The dimensions of the cowl lip are 15.2 by 3.8 by $0.64 \mathrm{~cm}$. However, for the finite element analysis only the $5-\mathrm{cm}$ central portion of the cowl lip was modeled to avoid end effects that would be difficult to quantify. The finite element mesh for this central portion was constructed by Melis and Gladden [1988]. The model was made up of 3294 solid, 8-noded hexagonal elements and has 4760 nodes. A large number of finite elements was required to deal with the severity of thermal loadings and gradients. Note from figure 6 that the temperature at a critical location of the component rises from 21 to $758^{\circ} \mathrm{C}$ in only $0.75 \mathrm{sec}$, indicating the severity of loading. However, because of the geometrical symmetry of the component, one 
need consider only half of the component for the finite element analysis, which results in a considerable savings in CPU time.

The steady-state temperature distribution in the component was obtained from Melis and Gladden [1988], which includes detailed results of a steady-state heat transfer analysis that was performed to obtain the steady-state temperature distribution in the cowl lip. Figure 6 shows this temperature distribution in the cowl lip. Figure 7 depicts the thermal loading cycle used in finite element analysis. The transient temperature distribution was obtained by using a linear interpolation technique. The highest temperatures in the component occur along the leading edge of the cowl lip. The temperature values thus obtained for the complete thermal loading cycle were used to perform the cyclic finite element analysis of the cowl lip.

\section{RESULTS AND DISCUSSION}

\subsection{Uniaxial Problems}

Figure 1 shows isothermal tensile stress-strain curves at different temperatures obtained by using the finite element implementation of Freed and Walker's viscoplastic model. The stress rate used in these computations is $0.0061 \mathrm{MPa} \mathrm{s}^{-1}$. Figure 1 also shows a comparison of curves obtained by Freed and Walker with those obtained experimentally. Good agreement exists among the curves obtained by Freed and Walker, by experiments, and by using the present implementation of the viscoplastic model.

The thermal and mechanical loadings used to generate the isothermal and nonisothermal hysteresis loops are shown in figure 2. These hysteresis loops for 
isothermal and nonisothermal loadings are shown in figures 3 and 4, respectively. The strain rates used in generating these isothermal and nonisothermal hysteresis loops are $0.001 \mathrm{~s}^{-1}$ and $1.5 \times 10^{-5} \mathrm{~s}^{-1}$, respectively. These figures also exhibit the hysteresis loops obtained by experiments. Again good agreement exists between the curves obtained experimentally and by using the finite element implementation. The difference in the values of stress obtained by using the finite element implementation and those observed in experiments (fig. 4), is due to the viscoplastic model. An error in the finite element implementation is not implied because the present results are in excellent agreement with the results of Freed and Walker [1993].

The good agreement between the calculated values from the finite element implementation and those obtained from experiments for the tensile curves and isothermal and nonisothermal hysteresis loops validates the finite element implementation of Freed and Walker's model. Such validation is a worthwhile investment of effort and computer time before applying the model to industrial structural engineering problems. These problems, in general, involve complex thermal/mechanical loadings in addition to their complex geometries and are, therefore, computationally intensive and time consuming.

\subsection{Cowl Lip Problem}

The most significant results of the cowl lip problem were obtained by plotting the stresses and strains along the leading edge, that is, the z-direction in figures 8 through 11 . The stress distribution in the z-direction at $0.75 \mathrm{~s}$ is exhibited in figure 8 , which shows the maximum (compressive) stress occurring 
at the leading edge of the cowl lip. Figure 9 displays the stress distribution in the z-direction of the cowl lip at $2.25 \mathrm{~s}$. A comparison of these figures reveals significant redistribution of stress in the component as a result of inelastic deformation. The stress along the leading edge is now seen to be tensile. This shows that viscoplastic models are capable of picking the redistribution of stress caused by inelastic deformation even for a short duration of $1.5 \mathrm{~s}$. Note that a nonunified elastic-plastic-creep analysis of this component performed by Arya et al. [1991] was unable to capture this redistribution of stress.

The strain distributions in z-direction in the cowl lip at $0.75 \mathrm{~s}$ and $2.25 \mathrm{~s}$ are shown in figures 10 and 11 , respectively. These figures show the values of total strain at different locations of the cowl lip. The compressive inelastic strain along the leading edge accumulates (increases) with time, which makes the total (tensile) strain along the leading edge decrease. This can be observed by comparing figures 10 and 11. For example, the total strain along the edge of the cowl lip reduces from a value of 0.00491 at $0.75 \mathrm{~s}$ to a value of 0.00483 at $2.25 \mathrm{~s}$.

The deformed shape of the segment at $0.75 \mathrm{~s}$ is plotted in figure 12. For easier examination, the displacements in this figure are magnified by a factor of 1000. This figure shows that the maximum deformation of the segment occurs near point $\mathbf{A}$. The component thickens in this region, which will lead to distortion of the coolant channel configuration. This thickening of the leading edge will also require more cooling down time, and this will lead to an early failure of the component. This indicates that, for the cowl lip problem, the parallel flow concept is not a particularly advantageous concept. 


\section{CONCLUSIONS}

A numerical solution methodology based on the finite element method is described for a viscoplastic model recently developed by Freed and Walker. It is illustrated for the finite element program MARC. However, the generality of methodology makes it easily adaptable to any other finite element program. The viability of methodology is demonstrated by applying it to several uniaxial problems and a multiaxial cowl lip problem. The results for uniaxial tensile and cyclic loadings obtained by using the finite element implementation show good agreement with the experimental results and with the results reported in the literature. The advantages of using an advanced viscoplastic model for nonlinear structural analyses are established by applying it to a multiaxial cowl lip problem and investigating the proposed parallel flow design concept. The results from the viscoplastic finite element analysis indicate that the parallel flow concept for the design of cowl lip is not a particularly advantageous concept, as it will lead to an early failure of the component. 


\section{REFERENCES}

ARYA, V.K., 1992, Nonlinear structural analysis of cylindrical thrust chambers using viscoplastic models. J. Propul. Power. 8, 3, 598-604.

ARYA, V.K., ARNOLD, S.M., 1992, Viscoplastic analysis of an experimental cylindrical thrust chamber liner. AIAA J. 30, 3, 781-789.

ARYA, V.K., KAUFMAN, A., 1989, Finite element implementation of Robinson's unified viscoplastic model and its application to some uniaxial and multiaxial problems. J. Eng. Comput. 6, 3, 237-247.

ARYA, V.K., et al., 1991, Finite element elastic-plastic-creep and cyclic life analysis of a cowl lip. Int. J. Fatigue Fract. Eng. Mater. Struct. 14, 10, 967-977.

CASSENTI, B.N., 1983, Research and development program for the development of advanced time-temperature dependent constitutive relationships, Vol. 1, Theoretical discussion. (R83-956011-1-2, United Technologies Research Center; NASA Contract NAS3-23273), NASA CR-168191.

FREED, A.D., WALKER, K.P., 1993, Viscoplasticity with creep and plasticity bounds. Int. J. Plast. 9, 2, 213-242.

MARC General Purpose Finite Element Program. MARC Analysis Research Corporation, Palo Alto, CA, Vols. A-D, 1992.

MELIS, M.E., GLADDEN, H.J., 1988, Thermostructural analysis with experimental verification in a high heat flux facility of a simulated cowl lip. Structures, Structural Dynamics and Material Conference, 29th Pt. 1. AIAA, New York, 106-115. 
WALKER, K.P., 1981, Research and development program for non-linear structural modeling with advanced time-temperature dependent constitutive relationships. (PWA-5700-50, United Technologies Research Center; NASA Contract NAS3-22055), NASA CR-165533. 
TABLE I.-MATERIAL CON-

STANTS FOR COPPER

[F\&W, 1993]

$\left[\mu=\mu_{0}+\mu_{1} \mathrm{~T} ; \mathrm{T}\right.$ is in degrees
Kelvin; $\mathrm{D}_{0}=\mathrm{C} / 100 ; \delta=0.035 ;$
\begin{tabular}{|c|c|c|}
\hline $\left.\mathrm{T}_{\mathrm{t}}=0.5 \mathrm{~T}_{\mathrm{m}} \cdot\right]$ & \\
\hline Constant & $\mathrm{Unit}$ & Value \\
\hline$\alpha$ & $\mathrm{K}^{-1}$ & $18 \times 10^{-6}$ \\
$\mu_{0}$ & $\mathrm{MPa}$ & 43000 \\
$\mu_{1}$ & $\mathrm{MPa} / \mathrm{K}$ & -17 \\
$\nu$ &.-- & 0.36 \\
$\mathrm{~A}$ & $\mathrm{~s}$ & $2 \times 10^{-1}$ \\
$\mathrm{C}$ & $\mathrm{MPa}$ & 13 \\
$\mathrm{f}$ & --- & 0.75 \\
$\mathrm{H}_{0}$ & --- & 20 \\
$\mathrm{~h}_{0}$ & $\mathrm{MPa}$ & 50 \\
$\mathrm{~h}_{\ell}$ & $\mathrm{MPa}$ & 15 \\
$\mathrm{~m}$ & --- & 0.5 \\
$\mathrm{n}$ & --- & 4.5 \\
$\mathrm{Q}$ & $\mathrm{J} / \mathrm{mol}$ & 200000 \\
$\mathrm{~T}_{\mathrm{m}}$ & $\mathrm{K}$ & 1356 \\
\hline
\end{tabular}




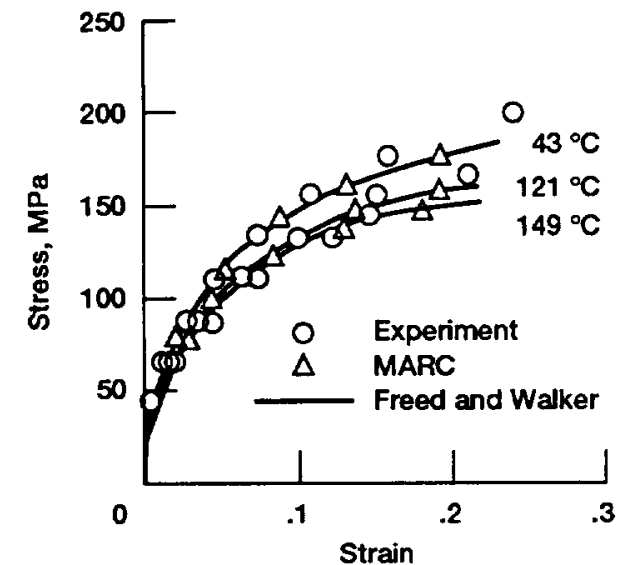

Figure 1.-Tensile stress-strain curves at different temperatures. (Stress rate is 0.0061 $\mathrm{MPa} / \mathrm{s}$.)

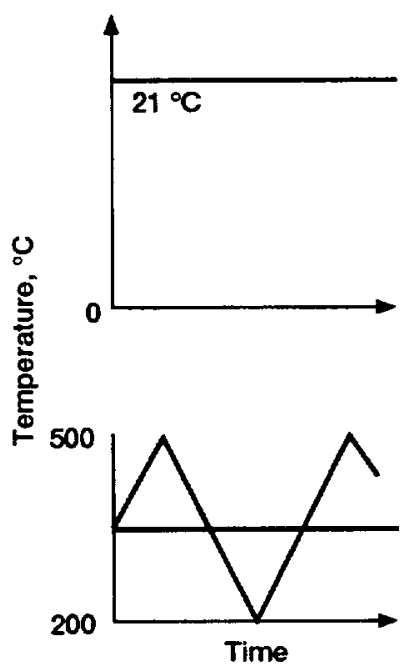

(a) Isothermal.

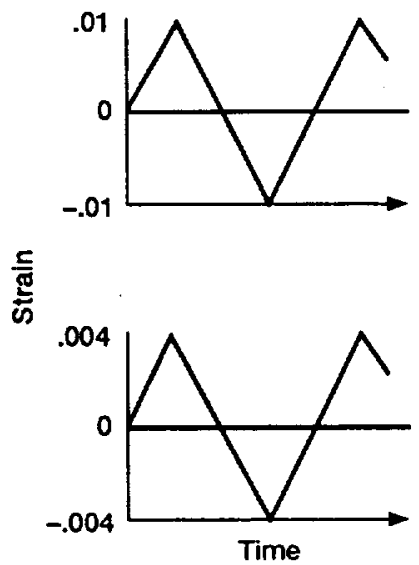

(b) Nonisothermal.
Figure 2.-Thermal and mechanical loadings used to generate hystersis loops by using the finite element implementation.

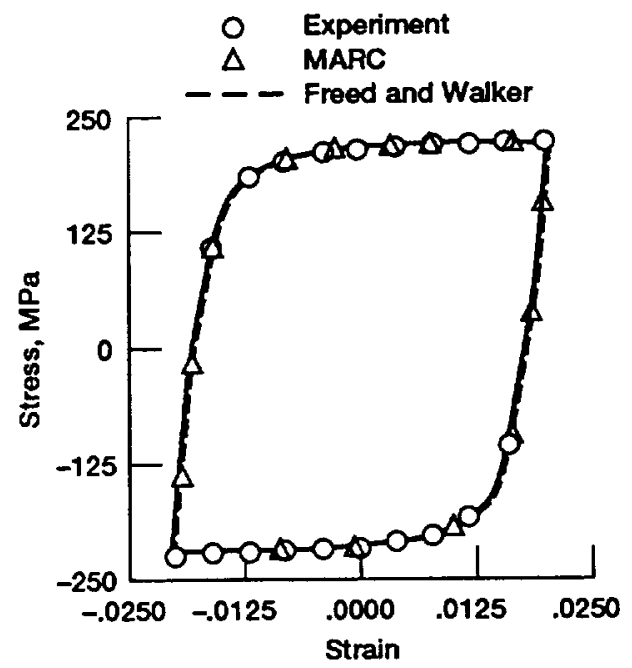

Figure 3.-Isothermal hysteresis loops at $21^{\circ} \mathrm{C}$. (Strain rate is $0.02 / \mathrm{s}$; strain range is 4 percent.)

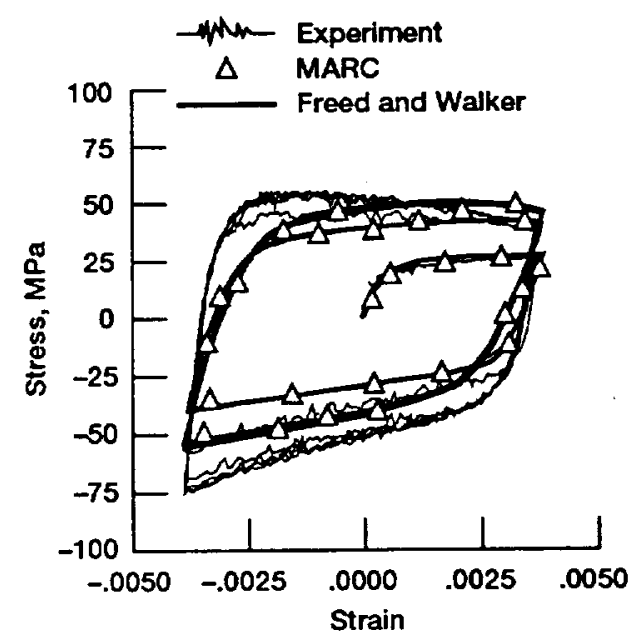

Figure 4.-Nonisothermal (in phase) hysteresis loops. (Temperature range is 200 to $500^{\circ} \mathrm{C}$; strain range is 0.0075 ; and strain rate is $1.5 \times 10^{-5} / \mathrm{s}$.) 


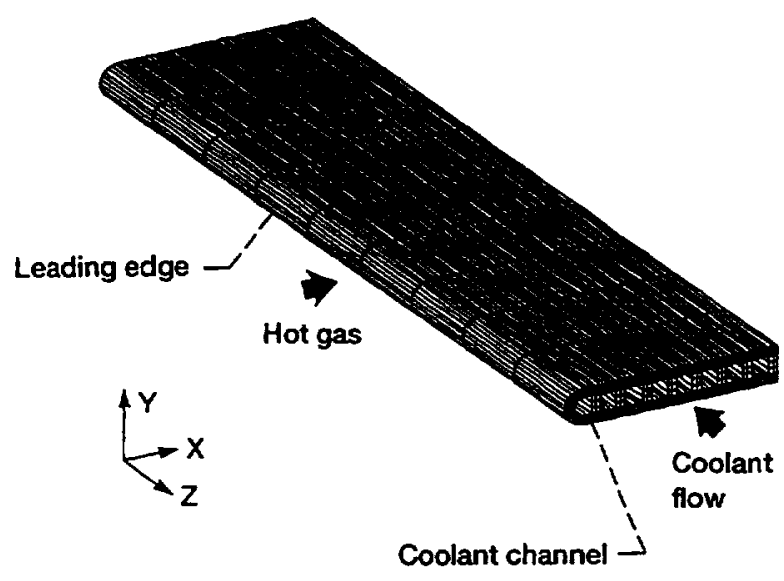

Figure 5.-Cowl lip finlte element model (3294 elements; 4760 nodes).

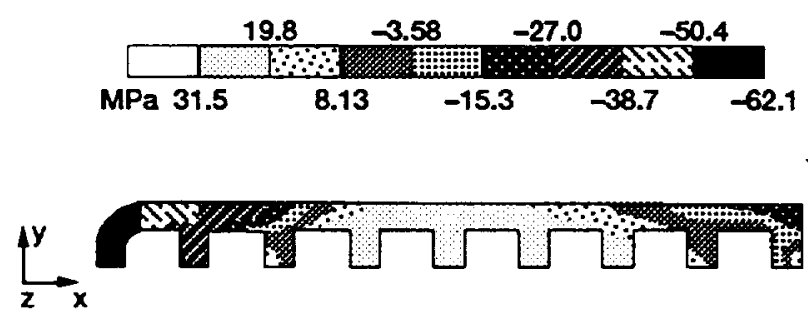

Figure 8.- Stress in the cowl lip at $0.75 \mathrm{~s} ; \mathrm{z}$-component.
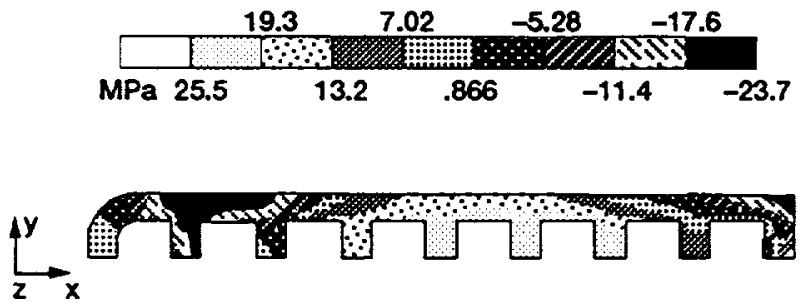

Figure 9. Stress in the cowl lip at $2.25 \mathrm{~s} ; \mathrm{z}$-component.

\begin{tabular}{|l|lllll}
\hline & .00459 & .00395 & .00331 & .00266 \\
\hline & 00491 & .00427 & .00363 & .00298 & .00234
\end{tabular}

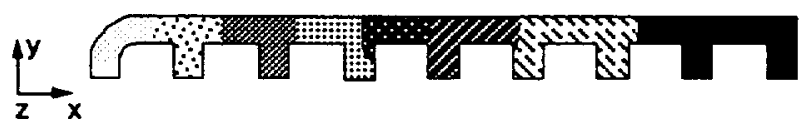

Figure 10.-Strain in the cowl lip at $0.75 \mathrm{~s} ; \mathrm{z}$-component.

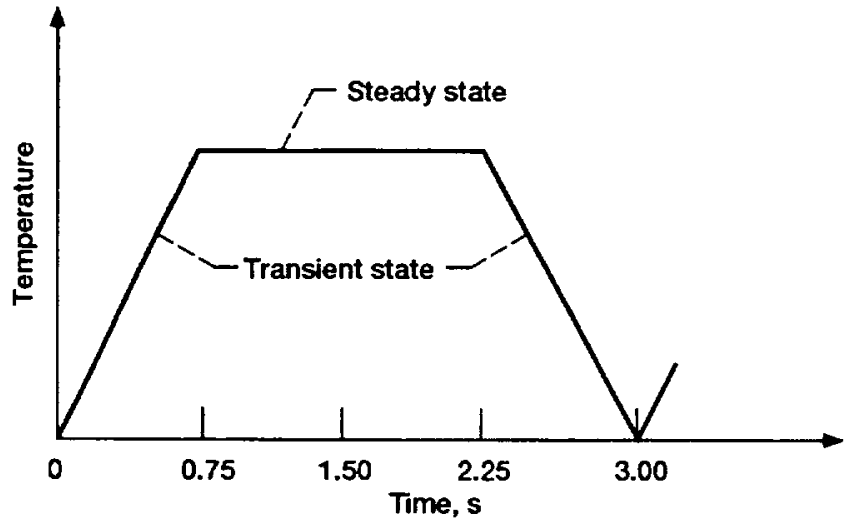

Figure 7.-Thermal loading cycle.

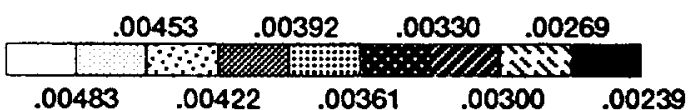

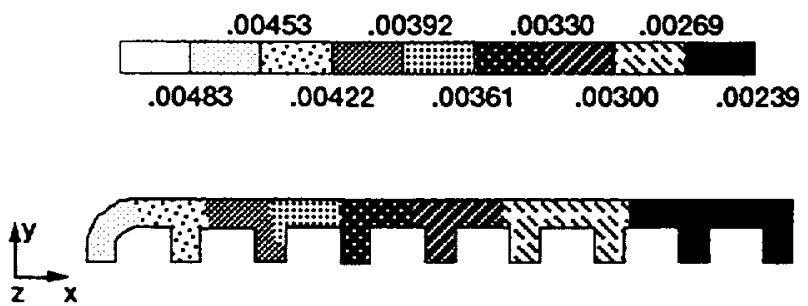

Figure 11.-Strain in the cowl lip at $2.25 \mathrm{~s} ; \mathrm{z}$-component.

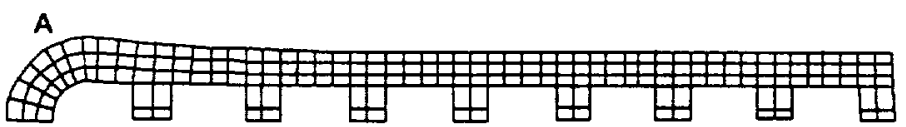

Figure 12.-Deformed shape of the segment at $0.75 \mathrm{~s}$. (For easier examination, this deformation is magnified by a factor of 1000.) 


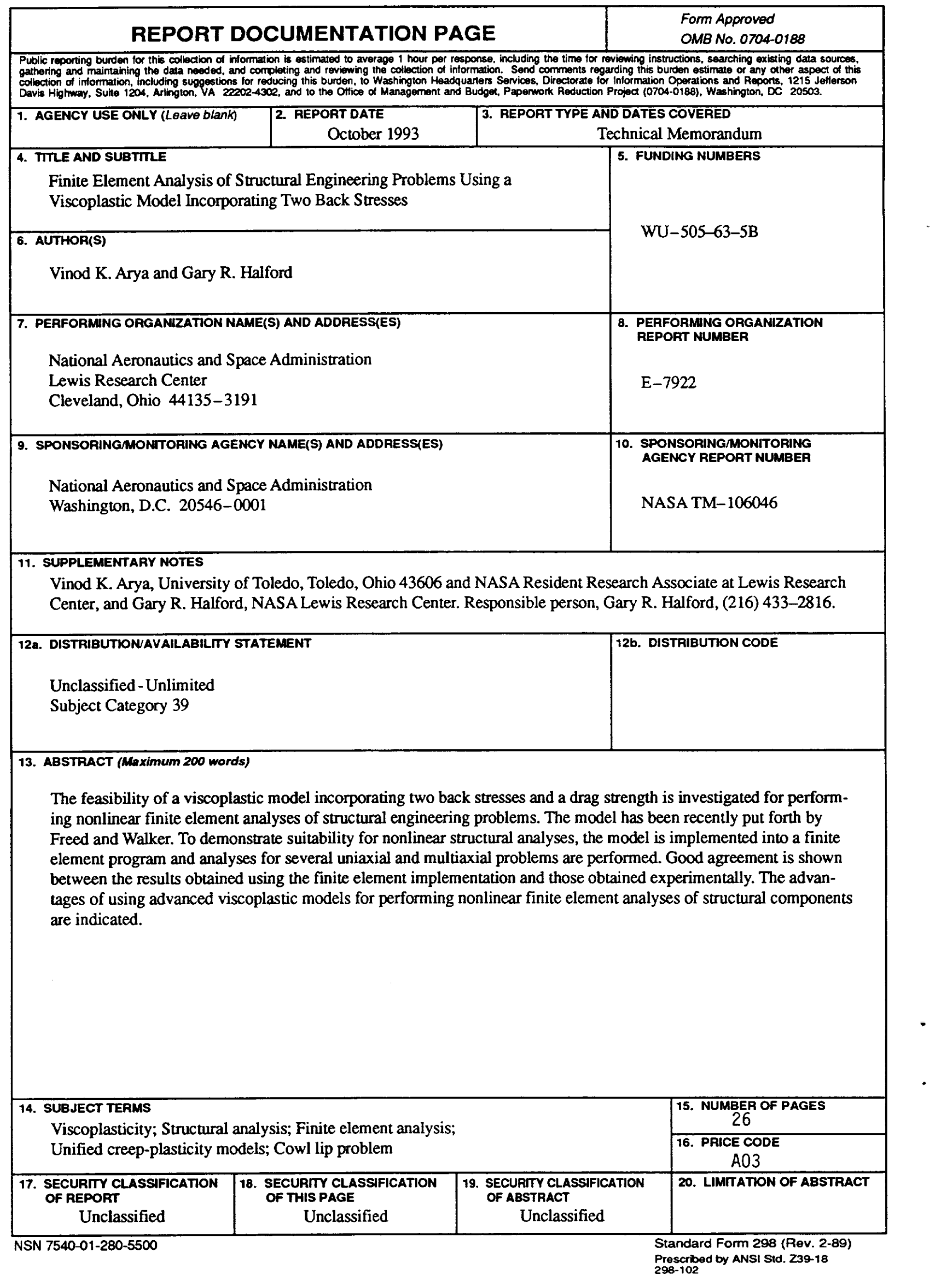

